\title{
Charged Rotating Black Branes in anti-de Sitter Einstein-Gauss-Bonnet Gravity
}

\author{
M. H. Dehghanif \\ Physics Department and Biruni Observatory, \\ Shiraz University, Shiraz 71454, Iran \\ and \\ Institute for Studies in Theoretical Physics and Mathematics (IPM) \\ P.O. Box 19395-5531, Tehran, Iran
}

We present a new class of charged rotating solutions in the Einstein-Gauss-Bonnet gravity with a negative cosmological constant. These solutions may be interpreted as black brane solutions with two inner and outer event horizons or an extreme black brane depending on the value of the mass parameter $m$. We also find that the Killing vectors are the null generators of the event horizon. The physical properties of the brane such as the temperature, the angular velocity, the entropy, the electric charge and potential are computed. We also compute the action and the Gibbs potential as a function of temperature and angular velocity for the uncharged solutions, and compute the angular momentum and the mass of the black brane through the use of Gibbs potential. We show that these thermodynamic quantities satisfy the first law of thermodynamics. We also perform a local stability analysis of the asymptotically AdS uncharged rotating black brane in various dimensions and show that they are locally stable for the whole phase space both in the canonical and grand-canonical ensemble. We found that the thermodynamic properties of Gauss-Bonnet rotating black branes are completely the same as those without the Gauss-Bonnet term, although the two solutions are quite different.

*Electronic address: dehghani@physics.susc.ac.ir 


\section{INTRODUCTION}

It is a general belief that Einstein's gravity is a low-energy limit of a quantum theory of gravity which is still unknown. Irrespective of the fundamental nature of quantum gravity, there should be a low-energy effective action which describes gravity at the classical level. This effective action will consist the Einstein-Hilbert action plus higher derivative (HD) terms. The appearance of HD gravitational terms can be seen, for example, in the renormalization of quantum field theory in curved spacetime [1], or in the construction of low-energy effective action of string theory [2].

Among the theories of gravity with higher derivative curvature terms, the Gauss-Bonnet gravity is of some special features in some sense. Indeed, in order to have a ghost-free action, the quadratic curvature corrections to the Einstein-Hilbert action should be proportional to the Gauss-Bonnet term [3, 4. This combination also appear naturally in the next-to-leading order term of the heterotic string effective action, and plays a fundamental role in ChernSimons gravitational theories [5]. From a geometric point of view, the combination of the Einstein-Gauss-Bonnet terms constitutes, for five dimensional spacetimes, the most general Lagrangian producing second order field equations, as in the four-dimensional gravity which the Einstein-Hilbert action is the most general Lagrangian producing second order field equations [6]. These facts provide a strong motivation for considering new exact solutions of the Einstein-Gauss-Bonnet gravity. Indeed, it is interesting to explore black holes in generalized gravity theories in order to discover which properties are peculiar to Einstein's gravity, and which are robust features of all generally covariant theories of gravity.

Due to the nonlinearity of the field equations, it is very difficult to find out nontrivial exact analytical solutions of Einstein's equation with the higher derivative terms. In most cases, one has to adopt some approximation methods or find solutions numerically. However, static spherically symmetric black hole solutions of the Gauss-Bonnet gravity have been found in Ref. [4. Black hole solutions with nontrivial topology in this theory have been also studied in Refs. [7, 8, 9]. The thermodynamics of charged static spherically symmetric black hole solutions has been considered in [10]. All of these known solutions are static. In this paper we will find the non-static asymptotically anti-de Sitter charged rotating black brane solutions in the Einstein-Gauss-Bonnet gravity.

The outline of our paper is as follows. We present a new class of charged rotating solutions 
with all rotation parameters for the Einstein-Gauss-Bonnet-Maxwell equation in Sec. [1]. In Sec. [II we consider the properties of these solutions in $(n+1)$ dimensions, and obtain their entropy, temperature, charge and electric potential. In Sec. IV, we compute the action, the angular momentum and the mass of the black brane by obtaining the Gibbs potential of the system. Section $\nabla$ will be devoted to the local stability analysis of the black brane in both the canonical and the grand-canonical ensemble. We finish our paper with some concluding remarks.

\section{CHARGED ROTATING SOLUTIONS OF EINSTEIN-GAUSS-BONNET'S EQUATION}

The gravitational action of an ( $n+1)$-dimensional asymptotically anti-de Sitter spacetimes with the Gauss-Bonnet term in the presence of an electromagnetic field can be written as

$$
I=-\frac{1}{16 \pi} \int d^{n+1} x \sqrt{-g}\left\{R+\frac{n(n-1)}{l^{2}}+\alpha\left(R_{\mu \nu \gamma \delta} R^{\mu \nu \gamma \delta}-4 R_{\mu \nu} R^{\mu \nu}+R^{2}\right)+F_{\mu \nu} F^{\mu \nu}\right\},
$$

where $F_{\mu \nu}=\partial_{\mu} A_{\nu}-\partial_{\nu} A_{\mu}$ is the electromagnetic tensor field, and $A_{\mu}$ is the vector potential. $\alpha$ is the Gauss-Bonnet coefficient with dimension (length $)^{2}$ and is positive in the heterotic string theory [⿴囗十 Einstein-Hilbert action with negative cosmological constant which are the leading ones while the Gauss-Bonnet term is the next-to-leading term in low-energy stringy effective action. Varying the action over the metric tensor $g_{\mu \nu}$ and electromagnetic field $F_{\mu \nu}$, the equations of gravitational and electromagnetic fields are obtained as

$$
\begin{gathered}
R_{\mu \nu}-\frac{1}{2} g_{\mu \nu} R+\frac{n(n-1)}{2 l^{2}} g_{\mu \nu}-\alpha\left\{\frac{1}{2} g_{\mu \nu}\left(R_{\gamma \delta \lambda \sigma} R^{\gamma \delta \lambda \sigma}-4 R_{\gamma \delta} R^{\gamma \delta}+R^{2}\right)\right. \\
\left.-2 R R_{\mu \nu}+4 R_{\mu \gamma} R_{\nu}^{\gamma}+4 R_{\gamma \delta} R_{\mu \nu}^{\gamma \delta}-2 R_{\mu \gamma \delta \lambda} R_{\nu}^{\gamma \delta \lambda}\right\}=T_{\mu \nu}, \\
\nabla_{\mu} F_{\mu \nu}=0,
\end{gathered}
$$

where $T_{\mu \nu}$ is the electromagnetic stress tensor

$$
T_{\mu \nu}=2 F_{\mu}^{\lambda} F_{\lambda \nu}-\frac{1}{2} F_{\lambda \sigma} F^{\lambda \sigma} g_{\mu \nu} .
$$

Equation (2) does not contain the derivative of the curvatures, and therefore the derivatives of the metric higher than two do not appear. Thus, the Gauss-Bonnet gravity is a special case of higher derivative gravity. In this section we want to obtain the rotating solutions of Eqs.(2)-(44). We first consider the metric with one rotation parameter. 


\section{A. Metric with one rotation parameter}

We assume the metric and the vector potential have the following forms

$$
\begin{aligned}
d s^{2} & =-f(r)(\Xi d t-a d \phi)^{2}+\frac{r^{2}}{l^{4}}\left(a d t-\Xi l^{2} d \phi\right)^{2}+\frac{d r^{2}}{f(r)}+r^{2} d \Omega^{2}, \\
\Xi^{2} & =1+\frac{a^{2}}{l^{2}} \\
A_{\mu} & =q h(r)\left(\Xi \delta_{\mu}^{0}-a \delta_{\mu}^{2}\right),
\end{aligned}
$$

where $d \Omega^{2}$ is the Euclidean metric on the $(n-2)$-dimensional submanifold with volume $\omega_{n-2}$. The functions $f(r)$ and $h(r)$ may be obtained by solving the field equations (2) and (3). This form of the metric was first introduced by Lemos [11] in four dimensions, and it has been generalized by Awad [12] for higher dimensions. Using Eq. (3) one can show that $h(r)=C_{1} / r^{n-2}$, where $C_{1}$ is an arbitrary real constant. To find the function $f(r)$, one may use any components of Eq. (2). The simplest equation is the $r r$-component of these equations which can be written as

$$
r^{5} f^{\prime}+2 r^{4} f-4 r^{6}+2 q^{2}-4 \alpha r^{3} f(r) f^{\prime}=0
$$

where the prime denotes a derivative with respect to the $r$ coordinate. The solution of Eq. (8) is

$f(r)=\frac{r^{2}}{2(n-2)(n-3) \alpha}\left\{1 \pm\left[1-4(n-2)(n-3) \alpha\left(\frac{1}{l^{2}}+\frac{(2 n-4) C_{1}^{2}}{(n-1) r^{2 n-2}}-\frac{C_{2}}{(n-1) r^{n}}\right)\right]^{1 / 2}\right\}$,

where $C_{2}$ is an arbitrary constant. As one can see from Eq. (9), the solution has two branches with " -" and "+" signs. In order to have an asymptotically anti-de Sitter spacetime, we should choose the branch with "-" sign. Indeed if one chooses the "-" sign, then the asymptotic behavior of the Ricci scalar for $\alpha=0$ is $-n(n+1) / l^{2}$, which is the case for

asymptotic AdS spacetimes, while the Ricci scalar for " +" sign branch diverges as $\alpha$ goes to zero. Choosing $C_{1}^{2}=(n-1) /(2 n-4) q^{2}$ and $C_{2}=(n-1) m$, then in the case of $\alpha=0$, one obtains the function $f(r)$ of Ref. [12 as expected. The thermodynamics and the no-hair theorem of the latter case in four dimension have been studied in Ref. [13, 14.

When $m$ and $q$ are zero, the vacuum solution is

$$
f(r)=\frac{r^{2}}{2(n-2)(n-3) \alpha}\left(1-\sqrt{1-\alpha \frac{4(n-2)(n-3)}{l^{2}}}\right) .
$$


Equation (10) shows that for a positive value of $\alpha$, this parameter should be less than $\alpha \leq l^{2} / 4(n-2)(n-3)$. Also note that the AdS solution of the theory has the effective cosmological constant

$$
l_{\text {eff }}^{2}=2(n-2)(n-3) \alpha\left(1-\sqrt{1-\frac{4(n-2)(n-3) \alpha}{l^{2}}}\right)^{-1} .
$$

\section{B. The generalized metric with all rotation parameters}

The rotation group in $(n+1)$-dimensions is $S O(n)$ and therefore the number of independent rotation parameters for a localized object is equal to the number of Casimir operators, which is $[n / 2] \equiv k$, where $[n / 2]$ is the integer part of $n / 2$. The generalization of the metric (5) with all rotation parameters is [12]

$$
\begin{aligned}
d s^{2}= & -f(r)\left(\Xi d t-\sum_{i=1}^{k} a_{i} d \phi_{i}\right)^{2}+\frac{r^{2}}{l^{4}} \sum_{i=1}^{k}\left(a_{i} d t-\Xi l^{2} d \phi_{i}\right)^{2} \\
& -\frac{r^{2}}{l^{2}} \sum_{i=1}^{k}\left(a_{i} d \phi_{j}-a_{j} d \phi_{i}\right)^{2}+\frac{d r^{2}}{f(r)}+r^{2} d \Omega^{2}, \\
\Xi^{2}= & \sum_{i=1}^{k}\left(1+\frac{a_{i}^{2}}{l^{2}}\right),
\end{aligned}
$$

where $d \Omega^{2}$ is now the Euclidean metric on the $(n-k-1)$-dimensional submanifold with volume $\omega_{n-k-1}$. Again one can show the asymptotically AdS solutions of Eqs. (22) and (32) are

$$
\begin{aligned}
f(r) & =\frac{r^{2}}{2(n-2)(n-3) \alpha}\left\{1-\sqrt{1-4(n-2)(n-3) \alpha\left(\frac{1}{l^{2}}-\frac{m}{r^{n}}+\frac{q^{2}}{r^{2 n-2}}\right)}\right\} \\
A_{\mu} & =\frac{(n-1)}{(2 n-4)} \frac{q}{r^{n-2}}\left(\left(\Xi \delta_{\mu}^{0}-a_{i} \delta_{\mu}^{i}\right), \quad(\text { no sum on } i) .\right.
\end{aligned}
$$

\section{PHYSICAL PROPERTIES OF THE SOLUTIONS}

As in the case of rotating black hole solutions of Einstein's gravity, the above metric given by Eqs. (11)-(13) has two types of Killing and event horizons. The Killing horizon is a null surface whose null generators are tangent to a Killing field. It is proved that a stationary black hole event horizon should be a Killing horizon in the four-dimensional Einstein gravity [15]. This proof can not obviously be generalized to higher order gravity, but the result is 
true for all the known static solutions. Although our solution is not static, the Killing vector,

$$
\chi=\partial_{t}+\sum_{i}^{k} \Omega_{i} \partial_{\phi_{i}}
$$

is the null generator of the event horizon. The event horizon define by the solution of $g^{r r}=f(r)=0$. The metric of Eqs. (11)-(13) has two inner and outer event horizons located at $r_{-}$and $r_{+}$, provided the mass parameter $m$ is greater than $m_{\text {crit }}$ given as

$$
m_{\text {crit }}=2\left(\frac{n-1}{n-2}\right)\left(\frac{n}{n-2}\right)^{-\frac{n-2}{2(n-1)}} l^{-\frac{n-2}{n-1}} q^{\frac{n}{n-1}} .
$$

In the case that $m=m_{\text {crit }}$, we will have an extreme black brane.

Analytical continuation of the Lorentzian metric by $t \rightarrow i \tau$ and $a_{i} \rightarrow i a_{i}$ yields the Euclidean section, whose regularity at $r=r_{+}$requires that we should identify $\tau \sim \tau+\beta_{+}$ and $\phi_{i} \sim \phi_{i}+i \beta_{+} \Omega_{i}$, where $\beta_{+}$and $\Omega_{i}$ 's are the inverse Hawking temperature and the angular velocities of the outer event horizon. One obtains

$$
\begin{aligned}
& \beta_{+}=\frac{4 \pi \Xi}{f^{\prime}\left(r_{+}\right)}=\frac{4 \pi \Xi l^{2} r_{+}^{2 n-3}}{n r_{+}^{2 n-2}-(n-2) l^{2} q^{2}}, \\
& \Omega_{i}=\frac{a_{i}}{\Xi l^{2}} .
\end{aligned}
$$

Usually entropy of the black holes satisfies the so-called area law of entropy which states that the black hole entropy equals to one-quarter of horizon area [16]. One of the surprising and impressive feature of this area law of entropy is its universality. It applies to all kind of black holes and black strings [17]. However, in higher derivative gravity the area law of entropy is not satisfied in general [18]. It is known that the entropy in the Gauss-Bonnet gravity is [19, 20]

$$
S=\frac{1}{4} \int d^{n-1} x \sqrt{\sigma}(1+2 \alpha \widetilde{R})
$$

where $\widetilde{R}$ is the Ricci scalar for the induced metric $\sigma_{a b}$ on the $(n-1)$-dimensional horizon. For our case $\widetilde{R}$ vanishes and therefore the entropy is equal to one-quarter of the area of the horizon as in the case of Einstein gravity, i.e.,

$$
S=(2 \pi)^{k} \omega_{n-k-1} \frac{\Xi}{4} r_{+}^{n-1},
$$

where $\omega_{n-1}=(2 \pi)^{k} \omega_{n-k-1}$ is the volume of the hypersurface at $t=$ const. and $r=$ const.

The charge of the black brane, $Q$, can be found by calculating the flux of the electric field at infinity, yielding

$$
Q=q \sqrt{\frac{(n-1)(n-2)}{2}}\left\{\Xi^{2}-\frac{a^{2}}{\alpha}\left(1-\sqrt{1-\frac{4(n-2)(n-3)}{l^{2}}}\right)\right\}^{1 / 2} \omega_{n-1} .
$$


The electric potential $\Phi$, measured at infinity with respect to the horizon, is defined by [21]

$$
\Phi=\left.A_{\mu} \chi^{\mu}\right|_{r \rightarrow \infty}-\left.A_{\mu} \chi^{\mu}\right|_{r=r_{+}},
$$

where $\chi$ is the null generator of the horizon given by Eq. (14). One finds

$$
\Phi=\sqrt{\frac{(n-1)}{2(n-2)}} \frac{q}{\Xi\left(r_{+}\right)^{n-2}} .
$$

\section{ACTION, ANGULAR MOMENTUM AND MASS OF THE UNCHARGED SOLUTIONS}

Now we turn to the calculation of the action and conserved quantities of the black brane in $(n+1)$ dimensions. In general the action given by Eq. (1) is divergent when evaluated on the solutions, as is the Hamiltonian, and other associated conserved quantities. One way of eliminating these divergences is to cut off the integral at a large $\mathcal{R}$ and subtract the solution with $m=q=0$ :

$$
\begin{aligned}
I_{\mathrm{reg}}= & -\frac{1}{16 \pi}\left\{\int d^{n+1} x \sqrt{-g}\left(R+\frac{n(n-1)}{l^{2}}\right)\right. \\
& \left.-\frac{f(r=\mathcal{R})}{f(r=\mathcal{R}, m=q=0)} \int d^{n+1} x \sqrt{-\widetilde{g}}\left(\widetilde{R}+\frac{n(n-1)}{l^{2}}\right)\right\} .
\end{aligned}
$$

The factor $f(r=\mathcal{R}) / f(r=\mathcal{R}, m=q=0)$ in front of the second integral is chosen such that the proper length of the circle which corresponds to the period $\beta_{+}$in the Euclidean metric at $r=\mathcal{R}$ coincides with each other in the two solutions [10]. Here we consider only the uncharged solutions, $q=0$. It is a matter of calculation to show that in the limit of $\mathcal{R} \rightarrow \infty$, the action is

$$
I_{\mathrm{reg}}=-\beta_{+0} \frac{\omega_{n-1}}{16 \pi} \frac{r_{+}^{n}}{l^{2}}=-\frac{\Xi \omega_{n-1}}{4 n} r_{+}^{n-1},
$$

where $\beta_{+0}$ denotes the value of $\beta_{+}$for $q=0$ which is

$$
T=\frac{1}{\beta_{+0}}=\frac{n r_{+}}{4 \pi \Xi l^{2}} .
$$

Using Eqs. (17) and (25) and the definition of Gibbs potential, $G\left(T, \Omega_{i}\right)=I_{\mathrm{reg}} / \beta_{+}$, one obtains:

$$
G\left(T, \Omega_{i}\right)=-\frac{\left(4 \pi l^{2}\right)^{n-1}}{4 n^{n}}\left(\frac{T}{\sqrt{1-l^{2} \sum_{i} \Omega_{i}^{2}}}\right)^{n} \omega_{n-1} .
$$


It is worthwhile to mention that $-(\partial G / \partial T)_{\Omega}$ gives the entropy, $S$, given in Eq. (19), as one expected. Also one may obtain the angular momentum of the black brane as

$$
J_{i}=-\left(\frac{\partial G}{\partial \Omega_{i}}\right)_{T, \Omega_{j}}=\frac{n}{16 \pi} \Xi m a_{i} \omega_{n-1}, \quad(j \neq i) .
$$

Now using the fact that the Gibbs function is the Legendre transformation of the energy $M(S, J)$ with respect to $S$ and $J$, we obtain

$$
M(S, J)=G\left(T, \Omega_{i}\right)+T S+\sum_{i} \Omega_{i} J_{i}=\frac{\omega_{n-1}}{16 \pi}\left(n \Xi^{2}-1\right) m
$$

One may note that the conserved and thermodynamic quantities obtained in this section are independent of the parameter $\alpha$, and therefore they are the same as those computed in the Einstein gravity.

\section{LOCAL THERMAL STABILITY OF THE UNCHARGED SOLUTIONS}

We first obtain the mass $M$ as a function of $S$, and $J$. Using the expressions (19), (27) and (28) for the entropy, the angular momentum, and the mass and the fact that $f\left(r_{+}\right)=0$, one obtains by simple algebraic manipulation

$$
M=\frac{\omega_{n-1}}{n} \frac{(n \Upsilon-1)}{\sqrt{\Upsilon(\Upsilon-1)}}
$$

where $\Upsilon$ is the real positive solution of the following equation:

$$
n^{2 n-2}(4 S)^{2 n}(\Upsilon-1)^{n-1}-\left(256 \pi^{2} l^{2} J^{2}\right)^{n-1} \Upsilon=0
$$

It is worthwhile to mention that the thermodynamic quantities calculated in Sec. $\square$ satisfy the first law of thermodynamics,

$$
d M=T d S+\sum_{i} \Omega_{i} d J_{i}
$$

Furthermore, by using Eqs. (17), (25) and (28), it is possible to obtain the equation of state for black brane in the Gauss-Bonnet gravity.

The local stability analysis in any ensemble can in principle be carried out by finding the determinant of the Hessian matrix $\left[\partial^{2} S / \partial X_{i} \partial X_{j}\right] \equiv \mathbf{H}_{X_{i}, X_{j}}^{S}$, where $X_{i}$ 's are the thermodynamic variables of the system. Indeed, the system is locally stable if the Hessian matrix 
satisfies $\mathbf{H}_{X_{i}, X_{j}}^{S} \leq 0$ [22. In our case the entropy $S$ is a function of the mass and the angular momentum, but the number of the thermodynamic variables depends on the ensemble which is used. In the canonical ensemble, the angular momentum is the fixed parameter, and for this reason the positivity of the thermal capacity $C_{J}$ is sufficient to assure the local stability. The thermal capacity $C_{J}$ at constant angular momentum is

$$
\left.C_{J}=T \frac{\partial S}{\partial T}=\frac{\Xi}{4}\left(\frac{(n-2) \Xi^{2}+1}{(n+2) \Xi^{2}-n-1}\right) r_{+}^{(} n-1\right) .
$$

As one can see from Eq. (32), $C_{J}$ is positive for all the allowed values of the metric parameters discussed in Sec. III, and therefore the asymptotically AdS rotating black branes in the canonical ensemble is locally stable.

In the grand-canonical ensemble, the thermodynamic variables are the mass and the angular momentum. Direct computation of the elements of the Hessian matrix of $S(M, J)$ with respect to $M$ and $J$ is a burdensome task, but the stability requirement $\mathbf{H}_{M, J}^{S} \leq 0$ may be rephrased as $\mathbf{H}_{S, J}^{M} \geq 0$ [23]. It is a matter of calculation to show that the black string is locally stable in the grand-canonical ensemble, since the determinant of Hessian matrix,

$$
\mathbf{H}_{S, J}^{M}=\frac{16}{l^{2} \Xi^{4}}\left(\frac{16 \pi}{n l \Xi^{2}}\right)^{k-1}\left[(n-2) \Xi^{2}+1\right]^{-1} r_{+}^{2-n(k+2)},
$$

is positive. It is worthwhile to note that $\alpha$ has not appeared in the thermodynamic quantities computed in this section. Thus, the uncharged solutions in the Gauss-Bonnet gravity has the same thermodynamic features as the solutions in the Einstein gravity. This fact is also true for the case of static spherically symmetric black holes with zero curvature horizon [7].

\section{CONCLUSIONS AND DISCUSSIONS}

We have found a new class of charged rotating solutions in Einstein theory with the GaussBonnet term and a negative cosmological constant. These solutions may be interpreted as black brane solutions with two inner and outer event horizons provided the mass parameter $m$ is greater than a critical value given by Eq. (15), and an extreme black brane for the critical value of the mass. We found that the Killing vectors are the null generators of the event horizon, and therefore the event horizon is a Killing horizon for the stationary solution of the Gauss-Bonnet gravity explored in this paper. The physical properties of the brane such as the temperature, the angular velocity, the entropy, the electric charge and potential have been computed. 
We also compute the regulated action of the uncharged solutions and found the Gibbs potential of the black brane in $(n+1)$ dimensions as a function of temperature and angular velocities. We obtained the entropy of the black brane by the well known formula, $S=$ $-(\partial G / \partial T)_{\Omega}$ and found out that it obeys the area the law of entropy. Of course this is in commensurate with the entropy formula of the holes in higher derivative gravity since the curvature of the horizon is zero for our case. Also the angular momentum and the mass of the brane are computed.

The first law of thermodynamics has been investigated for the uncharged rotating black brane and found that the thermodynamic quantities satisfy this law. Also the local stability of the asymptotically AdS uncharged rotating black brane both in the canonical and grandcanonical ensemble was investigated through the use of the determinant of the Hessian matrix of the mass with respect to its thermodynamic variables. We showed that the black branes in various dimensions are locally stable for the whole phase space both in the canonical and grand-canonical ensemble. We also found that thermodynamic properties of Gauss-Bonnet rotating black branes are completely the same as those without the Gauss-Bonnet term, although the two solutions are quite different. It seems that the thermodynamics of all the black holes/branes with zero curvature horizon are robust features of all generally covariant theories of gravity.

The computation of the action and conserved quantities through the use of AdS/CFT correspondence and also the stability analysis of the charged rotating black brane will be given elsewhere.

[1] N. D. Birrell and P. C. W. Davies, Quantum Fields in Curved Space (Cambridge University Press, Cambridge, England, 1982).

[2] M. B. Greens, J. H. Schwarz and E. Witten, Superstring Theory, (Cambridge University Press, Cambridge, England, 1987); D. Lust and S. Theusen, Lectures on String Theory, (Springer, Berlin, 1989); J. Polchinski, String Theory, (Cambridge University Press, Cambridge, England, 1998).

[3] B. Zwiebach, Phys. Lett. B 156, 315 (1985).

[4] D. G. Boulware and S. Deser, Phys. Rev. Lett., 55, 2656 (1985). 
[5] A. H. Chamseddine, Phys. Lett. B 233, 291 (1989); F. Muller-Hoissen, Nucl. Phys. B349, 235 (1990).

[6] D. Lovelock, J. Math. Phys. 12, 498 (1971); N. Deruelle and L. Farina-Busto, Phys. Rev. D 41, 3696 (1990); G. A. M. Marigan, ibid. 46, 4320 (1992); 4340 (1992); C. C. Briggs, gr-qc/9808050 (1998).

[7] R. Cai and K. Soh, Phys. Rev. D 59, 044013 (1999); R. Cai, ibid. 65, 084014 (2002).

[8] R. Aros, R. Troncoso and J. Zanelli, Phys. Rev. D 63, 084015 (2001).

[9] Y. M. Cho and I. P. Neupane, Phys. Rev. D 66, 024044 (2002).

[10] J. E. Lidsey, S. Nojiri and S. D. Odintsov, J. High Energy Phys., 06, 026 (2002); M. Cvetic, S. Nojiri, S. D. Odintsov, Nucl. Phys. B628, 295 (2002); S. Nojiri and S. D. Odintsov, Phys. Lett. B 521, 87 (2001); ibid. 523, 165 (2001).

[11] J. P. S. Lemos and V. T. Zanchin, Phys. Rev. D 54, 3840 (1996).

[12] A. M. Awad, hep-th/0209238.

[13] M. H. Dehghani, Phys. Rev. D 66, 044003, (2002).

[14] M. H. Dehghani and T. Jalali, Phys. Rev. D 66 (to be published).

[15] S. W. Hawking, Commun. Math. Phys. 25, 152 (1972); S. W. Hawking and G. F. R. Ellis, The Large Scale Structure of Spacetime (Cambridge University Press, 1973).

[16] J. D. Beckenstein, Phys. Rev. D 7, 2333 (1973); S. W. Hawking, Nature (London) 248, 30 (1974); G. W. Gibbons and S. W. Hawking, Phys. Rev. D 15, 2738 (1977).

[17] C. J. Hunter, Phys. Rev. D 59, 024009 (1999); S. W. Hawking, C. J. Hunter and D. N. Page, ibid. 59, 044033 (1999); R. B. Mann, ibid. 60, 104047 (1999); ibid. 61, 084013 (2000);

[18] D. L. Wiltshire, Phys. Lett. B 169, 36 (1986); Phys. Rev. D 38, 2445 (1988); M. Lu and M. B. Wise, ibid. 47, 3095,(1993); M. Visser, ibid. 48, 583 (1993); R. C. Myers, Nucl. Phys. B289, 701 (1993).

[19] T. Jacobson and R. C. Myers, Phys. Rev. Lett. 70, 3684 (1993); R. M. Wald, Phys. Rev. D 48, 3427, (1993); M. Visser, ibid. 48, 5697 (1993); V. Iyer and R. M. Wald, ibid. 50, 846 (1994).

[20] T. Jacobson, G. Kang and R. C. Myers, Phys. Rev. D 49, 6587,(1994); R. C. Myers, grqc/9811042.

[21] M. M. Caldarelli, G. Cognola and D. Klemm, Class. Quantum Grav. 17, 339 (2000).

[22] M. Cvetic and S. S. Gubser, J. High Energy Phys. 04, 024 (1999). 
[23] S. S. Gubser and I. Mitra, J. High Energy Phys., 08, 018 (2002). 